\title{
Relación entre índices aterogénicos y obesidad abdominal en pobladores del distrito de Trujillo, Perú
}

\author{
Relationship between atherogenic indices and abdominal obesity in residents of \\ Trujillo district, Peru
}

Carmen Yolanda Araujo Anticona 1,a, Jorge Luis Díaz-Ortega ${ }^{11, b}$, Rosa Patricia Gálvez Carrillo ${ }^{1, c}$

\section{Filiación y grado académico \\ 1 Universidad César Vallejo, Trujillo, Perú. \\ a Bachiller del Programa Académico de Nutrición. \\ b Doctor en Ciencias Biomédicas, docente del Programa Académico de Nutrición. Doctor en Ciencias Ambientales, docente del Programa Académico de Nutrición.}

(iD) ORCID iD de Carmen Araujo https://orcid.org/0000-0002-0281-517X

(iD) ORCID iD de Jorge Díaz-Ortega https://orcid.org/0000-0002-6154-8913

(iD) ORCID iD de Rosa Gálvez https://orcid.org/0000-0002-4612-109X

Contribución de autoría

Todos los autores contribuyeron en el diseño, ejecución, redacción y revisión del artículo.

Fuentes de financiamiento

Autofinanciado.

Conflictos de interés

Los autores declaran no tener conflictos de interés.

Recibido: 20-01-2021

Arbitrado por pares

Aceptado: 02-04-2021

Citar como

Araujo Anticona CY, Díaz-Ortega JL, Gálvez Carrillo RP. Relación entre índices aterogénicos y obesidad abdominal en pobladores del distrito de Trujillo, Perú. Rev Peru Cienc Salud. 2021; 3(2): 97-103. doi: https://doi. org/10.37711/rpcs.2021.3.2.311

Correspondencia

Jorge Luis Díaz Ortega

Dirección: Mz. I'Lote 1 Urb. Vista Hermosa Distrito de Trujillo, Departamento de la Libertad, Perú.

Email:jdiazo@ucv.edu.pe

\section{RESUMEN}

Objetivo. Determinar la relación entre los índices aterogénicos y la presencia de obesidad abdominal en pobladores del distrito de Trujillo, Perú. Los índices aterogénicos relacionados con la obesidad pueden brindar una información adicional al riesgo establecido por la obesidad. Métodos. El estudio fue de diseño no experimental, descriptivo correlacional y de corte transversal. La muestra estuvo conformada por 319 personas adultas cuyas edades oscilan entre 25 y 65 años que acudieron a cuatro centros de salud de la ciudad entre julio y diciembre de 2019. Para evaluar los índices aterogénicos se midieron las fracciones lipídicas de colesterol, triglicéridos, CHDL y cLDL mediante el uso de Colesterometro Mission ${ }^{\circledR}$. Con respecto a la presencia de obesidad abdominal se utilizaron los indicadores de la circunferencia de la cintura establecidos por la Asociación Latinoamericana de Diabetes. Resultados. El análisis estadístico empleado para relacionar ambas variables fue la prueba de chi cuadrado en el programa SPSS versión 25 . Se determinó que el 61,1\% de la población presenta obesidad abdominal. En cuanto a los índices aterogénicos, estos alcanzaron un diagnóstico de riesgo en el 75,5\% para el índice TG/cHDL; $67,4 \%$ en el índice de Castelli y 56,4\% para el índice CLDL/ cHDL. Solamente se encontró relación entre el índice TG/cHDL y la obesidad abdominal $(p=0,004)$. Conclusión. El índice TG/cHDL se asocia mejor con la obesidad abdominal y debe ser considerado adicionalmente en el diagnóstico probable o seguimiento de enfermedad coronaria o insulinorresistencia en el obeso.

Palabras clave: obesidad abdominal; colesterol; triglicéridos; Lipoproteínas HDL; enfermedades cardiovasculares (Fuente: DeCS-BIREME).

\section{ABSTRACT}

Objective. To determine the relationship between atherogenic indices and the presence of abdominal obesity in residents of the district of Trujillo, Peru. Atherogenic indices related to obesity can provide additional information to the risk established by obesity. Methods. The study was a non-experimental, descriptive correlational and cross-sectional design. The sample consisted of 319 adults between the ages of 25 and 65 years who attended four health centers in the city between July and December 2019. To evaluate the atherogenic indices, the lipid fractions of cholesterol, triglycerides, HDL-C and LDL-C were measured using the Mission Cholesterometer ${ }^{\circledast}$. Regarding the presence of abdominal obesity, the waist circumference indicators established by the Latin American Diabetes Association were used. Results. The statistical analysis used to relate both variables was the chi square test in the SPSS version 25 program. Abdominal obesity was found in $61.1 \%$ of the population. As for the atherogenic indexes, these reached a diagnosis of risk in $75.5 \%$ for the TG/HDL-C cholesterol index, $67.4 \%$ for the Castelli index and $56.4 \%$ for the LDL-C/HDL-C cholesterol index. Only a relationship was found between the TG/ HDL-C index and abdominal obesity $(p=0.004)$. Conclusion. The TG/HDL-C ratio is better associated with abdominal obesity and should be further considered in the probable diagnosis or follow-up of coronary heart disease or insulin resistance in the obese.

Keywords: abdominal obesity; cholesterol; triglycerides; HDL lipoproteins; cardiovascular diseases (Source: MeSH - NLM). 


\section{INTRODUCCIÓN}

La obesidad es considerada por diversos autores como un estado inflamatorio crónico debido a la acumulación excesiva de grasa corporal, ocasionando un cambio morfológico y funcional en los adipocitos, lo que además se asocia a otras patologías como dislipidemias, diabetes, hipertensión, problemas óseos y hasta cáncer, específicamente los de tipo gastrointestinal ${ }^{(1)}$.

En el Perú, según la información de la Encuesta Demográfica y de Salud Familiar-ENDES del año 2019, se ha encontrado que el $60,1 \%$ de personas de 15 y más años de edad padece de exceso de peso. En relación a la obesidad a nivel nacional, el 22,3\% son obesos, siendo mayor en mujeres, también en el área rural ${ }^{(2)}$. Entre los factores que favorecen el balance positivo de energía y ganancia de peso en la sociedad actual, tenemos al aumento progresivo del consumo per cápita de alimentos hipercalóricos; la disminución de la actividad física y mayor tendencia hacia actividades sedentarias como la observación de la televisión y el uso de dispositivos electrónicos ${ }^{(3)}$.

La enfermedad cardiovascular (ECV), especialmente la enfermedad arterial coronaria (EAC), sigue siendo la principal causa de morbimortalidad a nivel mundial, siendo un gran problema de salud pública tanto en países desarrollados como en vías de desarrollo y se caracteriza por la presencia de placas ateromatosas que disminuyen el aporte de oxígeno al corazón al interior de las arterias coronarias; condición que puede resultar en la reducción del flujo sanguíneo coronario, disminuyendo la llegada del oxígeno al corazón ${ }^{(4)}$.

El riesgo cardiovascular puede medirse mediante las concentraciones de lipoproteínas, por medio de un análisis de perfil lipídico (colesterol total, triglicéridos, colesterol de baja densidad (CLDL) y colesterol de alta densidad (cHDL). Las alteraciones de estas lipoproteínas representan el $50 \%$ de riesgo para su desarrollo. Adicionalmente al análisis del perfil lipídico convencional, se han propuesto tres índices de riesgo aterogénico: el índice de Castelli (colesterol total/cHDL), el índice de Kannel (cHDL/CLDL) y el índice TG/CHDL, debido a que estas aportan información acerca del balance entre lipoproteínas aterogénicas y antiaterogénicas; así, mientras más alto sea el valor de estos cocientes mayor será el riesgo cardiovascular ${ }^{(5)}$.

El cociente colesterol total/cHDL o índice de Castelli representa una alta capacidad predictiva de interpretación en el desarrollo de riesgo coronario en comparación a si se emplea el colesterol total y cHDL de forma independiente, brindándonos un panorama sobre si los valores de CHDL son suficientes para manejar la carga total del colesterol (6). El aumento de este cociente hace referencia a un riesgo elevado de desarrollar enfermedad cardiovascular porque normalmente está asociada con el estado inflamatorio de las placas de ateroma y al grosor íntima-media de la arteria carótida ${ }^{(5,6)}$.

El Cociente cLDL/ CHDL o índice de Kannel se trata de un cociente muy parecido al anterior, pero que no se puede utilizar en los casos de hipertrigliceridemia $>300$ $\mathrm{mg} / \mathrm{dl}$, debido a que la mayor parte del contenido de colesterol se encontraría en la fracción de la lipoproteína de muy baja densidad (VLDL) y, por lo tanto, es preferible utilizar el índice de Castelli ${ }^{(5)}$. Este índice está relacionado con la disfunción del endotelial, siendo factor principal en la patogenia de la ateroesclerosis ${ }^{(7)}$. El cociente CLDL/ $\mathrm{CHDL}$, es un predictor eficaz del riesgo cardiovascular, sobre todo en las personas con triglicéridos elevados. El mayor riesgo se observó en personas con un cociente $\mathrm{cLDL} / \mathrm{cHDL}>5$ y triglicéridos $>200 \mathrm{mg} / \mathrm{dl}^{(8)}$.

El índice o cociente TG/CHDL relaciona concentraciones triglicéridos y $\mathrm{CHDL}$; el aumento de este cociente funciona como un indicador indirecto del tamaño de las partículas de CLDL y nos indica un mayor número de partículas de CLDL pequeñas y densas, las cuales se relacionan con el inicio y progresión de la aterosclerosis ${ }^{(9,10)}$. Asimismo, en algunas investigaciones los tres indicadores aterogénicos se les ha identificado como predictores del síndrome metabólico ${ }^{(7,11)}$.

La valoración de los índices aterogénicos ayuda a establecer medidas preventivas y el tratamiento adecuado en personas, principalmente con sobrepeso u obesidad, que no presentan síntomas, pero con un elevado riesgo de padecer enfermedades cardiometabólicas, ya que reflejan mejor el desbalance de las lipoproteínas aterogénicas y antiaterogénicas.

Por consiguiente, el objetivo general de esta investigación fue determinar la relación de los índices aterogénicos con la obesidad abdominal en pobladores del distrito de Trujillo, 2019. Entre los objetivos específicos se consideró determinar el nivel del índice de Castelli (CT/CHDL); índice de Kannel (cLDL/cHDL); e índice TG/ $\mathrm{CHDL}$; para finalmente evaluar la presencia de obesidad abdominal y su vinculación con cada uno de los índices aterogénicos.

\section{MÉTODOS}

\section{Tipo y diseño de investigación}

El presente estudio de investigación, según el fin que se persigue, fue de tipo observacional, correlacional y transversal. 


\section{Población y muestra}

La población estuvo constituida por los pobladores adultos del distrito de Trujillo, provincia de Trujillo, departamento de La Libertad (Perú), con edades entre los 25 y los 65 años. Se utilizó la fórmula para población infinita y se consideró una prevalencia del $22,3 \%$ de obesidad en población adulta según la ENDES del $2018^{(2)}$ y un error del $4,57 \%$, obteniéndose una muestra de 319 personas. La selección de las personas fue por conveniencia de 04 establecimientos de salud de la Microred-Trujillo (Hospital de Especialidades Básicas La Noria y Centros de Salud Los Jardines, San Martín y Sagrado Corazón) durante el periodo de julio a diciembre del 2019. Como criterios de exclusión se consideró a aquellas personas con dificultades de raciocinio, alguna discapacidad física, enfermedades crónicas degenerativas como: cáncer, diabetes, enfermedades cardiovasculares y enfermedades respiratorias crónicas o tratamiento farmacológico para las dislipidemias.

\section{Instrumentos de recolección de datos}

Se utilizaron como instrumentos mecánicos-objetivos: Una cinta métrica metálica Cescorf para medir la circunferencia de cintura, colesterometro Mission ${ }^{\circledR}$ para la evaluación del perfil lipídico: colesterol total, CLDL, CHDL y TG, valores que se utilizaron para calcular el índice de Castelli, índice de Kannel y índice TG/cHDL.

\section{Evaluación de los índices aterógenicos}

Se realizaron coordinaciones previas con los jefes de los establecimientos de salud seleccionados para el uso de los ambientes generalmente en el consultorio de Nutrición con cronogramas establecidos y en horario de 7:00 a 10:00 am; asi como comunicaciones previas a las personas que acudían al hospital sobre las evaluaciones gratuitas del perfil lipídico a realizar, sugiriendo su asistencia en ayunas.

La muestra sanguínea de cada participante fue extraída del dedo índice, pinchando con lanceta; la primera gota fue desechada y la muestra se tomó a partir de la segunda gota hasta llenar la medida indicada en la micropipeta correspondiente a $30 \mu \mathrm{L}$. Luego la sangre se depositó sobre la tira reactiva previamente colocada en el colesterometro, registrando la lectura del colesterol total, $\mathrm{CHDL}, \mathrm{CLDL}$, TG y el índice de Castelli. El índice de Kannel (cLDL/cHDL) y el índice TG/cHDL se calcularon a través de los cocientes entre los valores del perfil lipídico involucrados. Se comparó los resultados junto a los valores recomendados y en riesgo para la prevención primaria indicado por Millan ${ }^{(5)}$ : Para índice de Castelli, los valores recomendables en hombres fueron $<4,5$ en mujeres $<4$; en riesgo cardiovascular en hombres y mujeres >
4,5 y 4, respectivamente, en el índice de Kannel fueron considerados valores recomendables para hombres < 3 y para mujeres $<2,5$; y para riesgo cardiovascular, en hombres y mujeres $\geq 3$ y $\geq 2,5$ respectivamente. Finalmente, para TG/HDL se consideró como valor para tendencia a resistencia a la insulina $>3^{(12)}$.

\section{Evaluación antropométrica}

Para la medición antropométrica de la circunferencia de cintura se utilizó una cinta métrica metálica "Cescorf". La persona permaneció de pie con los pies juntos, el torso desnudo y los brazos relajados a cada lado de su cuerpo. Se colocó la cinta métrica alrededor del cuerpo, específicamente desde el punto medio entre la última costilla y la cresta iliaca, se tomó la medición al final de una espiración normal. Según los siguientes indicadores para la circunferencia de cintura según el consenso de la Asociación Latinoamericana de Diabetes (ALAD) ${ }^{(13)}$ : normal para hombres $<94 \mathrm{~cm}$ y mujeres $<88 \mathrm{~cm}$; obesidad abdominal hombres $\geq 94 \mathrm{~cm}$ y mujeres $\geq 88 \mathrm{~cm}$.

\section{Análisis de datos}

Con los datos obtenidos mediante la ficha de recolección de datos, para el procesamiento de datos se utilizó el programa Microsoft Excel 2016 y para el análisis de los datos se empleó el programa estadístico Statistical Package for the Social Sciencies (SPSS) versión 25. Se determinó la relación de ambas variables mediante la prueba de chi cuadrado con un nivel de significancia de $p<0,05$.

\section{Aspectos éticos}

Para la realización de la investigación se consideró la Declaración de Helsinki de la Asociación Médica Mundial ${ }^{(14)}$, a fin de poder garantizar la privacidad y confidencialidad de la persona partícipe.

Las personas fueron informadas sobre sus derechos, procedimientos del estudio a seguir y beneficios en caso de que aceptaban participar o no en la investigación y el respeto a la privacidad; de modo que las identidades de los participantes no serán divulgadas ni utilizadas para otros fines ajenos al estudio de acuerdo a la Ley N. ${ }^{\circ}$ $29733^{(15)}$ que hace referencia a la ley de protección de datos personales. Finalmente, se les solicitó la firma del consentimiento de su participación en la investigación.

\section{RESULTADOS}

En la tabla 1 se observó que de los 319 (100,0\%) adultos del distrito de Trujillo, $195(61,1 \%)$ presentaron obesidad abdominal y 124 (38,9\%) no presentaron obesidad abdominal. 
Tabla 1. Presencia de obesidad abdominal en pobladores del distrito de Trujillo, 2019

\begin{tabular}{lcc}
\hline Obesidad & $\mathbf{f i}$ & $\%$ \\
\hline Sí & 195 & 61,1 \\
No & 124 & 38,9 \\
Total & 319 & 100,0 \\
\hline
\end{tabular}

En la tabla 2 se observó que los índices aterogénicos en los pobladores adultos del distrito de Trujillo alcanzaron diagnóstico de riesgo cardiovascular, observándose para el índice TG/cHDL, índice de Castelli y cLDL/cHDL, valores del $75,5 \%$ (241); $67,4 \%$ (215) y $56,4 \%$ (180), respectivamente.

En la tabla 3 se observó que las personas con valores recomendables del índice de Castelli, el 57,7 \% (60) presentaron obesidad abdominal. En cuanto a las personas que presentaron valores de riesgo del índice de Castelli, el $62,8 \%(135)$ eran obesos. Por lo tanto, al predominar la obesidad en las personas, ya sea con diagnóstico de Índice de Castelli normal y de riesgo, no se observó asociación entre el índice de Castelli y la presencia de obesidad $(p=0,381)$.

En la tabla 4 se muestra que de las personas con valores recomendables de índice de Kannel, el 58,3\% (81) presentaron obesidad abdominal en tanto que de las personas que presentaron valores de riesgo de índice de Kannel, el 63,3 \% (114) fueron obesos. De esta manera, el índice de Kannel no se asoció con la presencia de la obesidad $(p=0,358)$.

En la tabla 5 se observó que de los pobladores adultos del distrito de Trujillo con valores recomendables del índice TG/cHDL, el 52,6\% (41) no presentaron obesidad abdominal, en tanto que las personas que presentaron valores de riesgo del índice TG/ cHDL, el 65,6 \% (158) eran obesos, existiendo de esta manera una relación muy significativa entre el índice de TG/cHDL y la presencia de obesidad $(p<0,01)$.

Tabla 3. Relación entre el índice de Castelli y la presencia de obesidad abdominal en pobladores del distrito de Trujillo, 2019

\begin{tabular}{lccccc}
\hline $\begin{array}{l}\text { Índice de Castelli } \\
\text { No presenta }\end{array}$ & \multicolumn{2}{c}{ Obesidad Abdominal } & Total & fi (p) \\
\cline { 2 - 4 } & No presenta & Presenta & & \\
\hline \multirow{2}{*}{ Recomendable } & N. ${ }^{\circ}$ & 44 & 60 & 104 & \\
& $\%$ & 42,3 & 57,7 & 100,0 & 0,381 \\
\multirow{2}{*}{ Riesgo } & N. ${ }^{\circ}$ & 80 & 135 & 215 & \\
& $\%$ & 37,2 & 62,8 & 100,0 & \\
\hline
\end{tabular}

Valor "p" obtenido según prueba de contingencia chi cuadrado.
Tabla 2. Índices aterogénicos en pobladores del distrito de Trujillo, 2019

\begin{tabular}{|c|c|c|c|c|c|c|}
\hline \multirow{2}{*}{ Diagnóstico } & \multicolumn{2}{|c|}{$\begin{array}{c}\text { Índice de } \\
\text { Castelli }\end{array}$} & \multicolumn{2}{|c|}{$\mathrm{cLDL} / \mathrm{cHDL}$} & \multicolumn{2}{|c|}{ TG/cHDL } \\
\hline & $\mathrm{fi}$ & $\%$ & fi & $\%$ & $\mathrm{fi}$ & $\%$ \\
\hline Recomendable & 104 & 32,6 & 139 & 43,6 & 78 & 24,5 \\
\hline Riesgo & 215 & 67,4 & 180 & 56,4 & 241 & 75,5 \\
\hline Total & 319 & 100,0 & 319 & 100,0 & 319 & 100,0 \\
\hline
\end{tabular}

\section{DISCUSIÓN}

La circunferencia de la cintura proporciona información en relación a la adiposidad abdominal, lo cual está asociado con la morbimortalidad de las enfermedades cardiovasculares y la insulinorresistencia ${ }^{(16)}$. Según esta medición, ha sido de gran utilidad para clasificar a la población y poder detectar quienes presentan o no obesidad abdominal; así pues, un alto porcentaje $(61,1 \%)$ de pobladores que fueron evaluados presentan obesidad abdominal, aunque en el estudio de Coronel ${ }^{(17)}$ en pacientes adultos de la ciudad de Jaén se evidenció un mayor porcentaje, del $74,13 \%$. Por el contrario, en la investigación de Castrillón ${ }^{(18)}$ en Lima, el 43,2 \% de los pobladores presenta obesidad abdominal, lo que indica un porcentaje menor del total de la muestra.

La obesidad abdominal identificada por la circunferencia de la cintura es considerada como un factor de riesgo para las enfermedades cardiovasculares, asociada con el tejido adiposo visceral que rodea a los órganos internos con mayor tamaño y número, tienen mayor actividad lipolítica. Esto ocasiona la liberación de ácidos grasos libres a la circulación portal donde ejerce acciones metabólicas adversas, sobre todo en el hígado, los macrófagos son activados por estos ácidos grasos, ocasionando una respuesta inflamatoria en el tejido adiposo hipertrófico y produciendo una mayor cantidad de citoquinas proinflamatorias; lo que facilita la

Tabla 4. Relación entre el índice de Kannel y la presencia de obesidad abdominal en pobladores del distrito de Trujillo, 2019

\begin{tabular}{lccccc}
\hline \multirow{2}{*}{$\begin{array}{l}\text { Índice de Kannel } \\
\text { No presenta }\end{array}$} & \multicolumn{3}{c}{ Obesidad Abdominal } & Total & fi (p) \\
\cline { 2 - 4 } Recomendable & N. ${ }^{\circ}$ & 58 & 81 & 139 & \\
\hline & $\%$ & 41,7 & 58,3 & 100,0 & 0,358 \\
Riesgo & N. ${ }^{\circ}$ & 66 & 114 & 180 & \\
& $\%$ & 36,7 & 63,3 & 100,0 & \\
\hline
\end{tabular}

Valor" $p$ " obtenido según prueba de contingencia chi cuadrado. 
Tabla 5. Relación entre el índice TG/CHDL y la presencia de obesidad abdominal en pobladores del distrito de Trujillo, 2019

\begin{tabular}{|c|c|c|c|c|c|}
\hline \multirow{2}{*}{$\begin{array}{l}\text { Índice TG/cHDL } \\
\text { No presenta }\end{array}$} & & \multicolumn{2}{|c|}{ Obesidad Abdominal } & \multirow{2}{*}{ Total } & \multirow{2}{*}{$f i(p)$} \\
\hline & & No presenta & Presenta & & \\
\hline \multirow{2}{*}{ Recomendable } & N.o & 41 & 37 & 78 & \multirow{4}{*}{$0,004^{*}$} \\
\hline & $\%$ & 52,6 & 47,4 & 100,0 & \\
\hline \multirow{2}{*}{ Riesgo } & N. ${ }^{\circ}$ & 83 & 158 & 241 & \\
\hline & $\%$ & 34,4 & 65,6 & 100,0 & \\
\hline
\end{tabular}

$p<0,01$ muy significativo según prueba de contingencia chi cuadrado.

aparición de la patogénesis de la disfunción endotelial que contribuye al desarrollo de la aterosclerosis ${ }^{(19,20)}$.

Según la Fundación Española del Corazón se recomienda medir la circunferencia abdominal ya que esta se presenta como principal factor de riesgo cardiovascular debido a la acumulación de grasa visceral a nivel abdominal. A medida que se acumula grasa visceral en el abdomen, el vientre se vuelve visiblemente más prominente, asociándose con anormalidades metabólicas ${ }^{(21)}$. Sin embargo, este parámetro debería ir acompañado con un indicador lipídico ideal que permita asegurar un mejor diagnóstico de riesgo cardiovascular.

La elevada proporción de diagnóstico de riesgo observado en los índices aterogénicos en los pobladores adultos de la Ciudad de Trujillo han sido reportados también por Berrocal y Torres ${ }^{(22)}$ en pacientes de la ciudad Lima, siendo índice de Castelli e índice cLDL/ CHDL elevados en el 49,7 y $51,4 \%$ respectivamente. Estos índices aportan información valiosa acerca del balance entre lipoproteínas aterogénicas y antiaterogénicas; así, mientras más alto el valor de estos cocientes mayor se supone el riesgo cardiovascular. El estudio de Millán et al. ${ }^{(5)}$ en Madrid hace referencia a la similitud entre ambos índices: $\mathrm{CT} / \mathrm{CHDL}$ y $\mathrm{CLDL} / \mathrm{CHDL}$, lo cual se explica porque aproximadamente dos tercios de colesterol en plasma se encuentra en las lipoproteínas de baja densidad (LDL); por lo tanto, existe un vínculo estrecho entre el CT y el $C L D L$, pudiendo aumentar su poder predictivo en la enfermedad cardiovascular y siendo el riesgo mayor en pacientes que presenten hipertrigliceridemia. La relación TG/CHDL da un panorama del tamaño de partículas del CLDL pequeñas y densas, facilitando la detección de un riesgo aterogénico aumentando. Además, en el estudio de Gonzales ${ }^{(11)}$ en Venezuela evidenció que el índice TG/ cHDL, según el análisis bioquímico, fue un indicador con alta capacidad predictiva para el síndrome metabólico debido a su principal característica, la dislipidemia aterogénica; aspecto a tomar en cuenta de tal presencia en la mayoría de los adultos que participaron en la presente investigación.
El no haber encontrado relación entre el índice de Castelli y la presencia de obesidad abdominal se debe principalmente a la forma de correlación, al hacer al índice de Castelli dicotómico; razón por la cual no coincide con la investigación de Coronel ${ }^{(17)}$ en el 2019, quien observó la relación proporcional del índice de Castelli con el IMC y no con el perímetro abdominal. Rosas et al. ${ }^{(23)}$, por su parte, observaron una tendencia creciente de las medias de los valores séricos de la relación CT/HDL-C $(p<0,001)$, conforme aumentaba el IMC. Sin embargo, es importante precisar su interpretación en las personas, ya que una concentración baja de colesterol puede estar en riesgo de enfermedad cardiovascular si presentan $\mathrm{CHDL}$ bajo $y$, por el contrario, con niveles de colesterol alto y $\mathrm{CHDL}$ alto no desarrollan problemas cardiovasculares por la función antiaterogénica del transporte reverso del colesterol ejercido por el $\mathrm{CHDL}^{(24)}$; aspecto que debe considerarse desde el sobrepeso. Precisamente este índice en valores elevados está asociado con inflamación e inestabilidad de las placas de ateromas y como predictor del grosor íntima-media de la arteria carótida ${ }^{(25)}$.

En cuanto al índice de Kannel (cLDL/cHDL), ya sea con valores recomendables y no recomendables, hubo un predominio de la obesidad abdominal, siendo contrario a los resultados de Domingo ${ }^{(7)}$, quien encontró relación significativa $(p<0,0001)$.

Por consiguiente, se puede observar que en las personas que presentan obesidad abdominal existe también una mayor proporción de presencia de índice aterogénico en riesgo. Cuando el índice da como resultado límites no deseables, esto indica que la persona presenta un desequilibrio entre ambas lipoproteínas; en este caso, si el colesterol es direccionado por las lipoproteínas aterogénicas y las cardioprotectoras como en el caso del $C H D L$, nos señala que puede deberse al aumento del CLDL o disminución del CHDL. Es probable que la no relación del índice de Kannel y la obesidad, se deban a una subestimación del colesterol total por el índice de Kannel, principalmente en obesos con hipertrigliceridemias por encima de $300 \mathrm{mg} / \mathrm{dL}^{(5)}$, que podrían estar concentrados en las partículas de VLDL-c, aspecto que no se tomó en cuenta en el presente estudio y que tomados los datos de manera transversal puede conllevar a posibles sesgos de análisis ${ }^{(26)}$.

En cuanto al índice $\mathrm{TG} / \mathrm{CHDL}$, este presenta una relación directa con la obesidad abdominal con resultado concordante con el estudio de Roa ${ }^{(27)}$ en Venezuela, con $p$ $<0,001$; así como con la investigación de Domingo ${ }^{(12)}$ en Madrid, aunque con $p<0,0001$. Se conoce el efecto de la obesidad central en el aumento de la resistencia insulínica 
y el impacto que esto tiene en el metabolismo de los lípidos, trayendo como consecuencia el incremento de los factores de riesgo para la enfermedad cardiovascular, ocasionando una respuesta inflamatoria y produciendo una mayor cantidad de citoquinas proinflamatorias; lo que contribuye al desarrollo de la ateroesclerosis ${ }^{(28)}$. Se ha determinado, además, que la relación TG/cHDL puede usarse para identificar de manera indirecta la resistencia a la insulina ${ }^{(29-33)}$ y el riesgo cardiometabólico ${ }^{(34,35)}$. Por consiguiente, es probable que principalmente los participantes obesos en el presente estudio estén desarrollando resistencia a la insulina, aspecto que no se delimitó en el mismo por ser costoso y por el tiempo para desarrollar su medición como es el caso de la utilización del índice Homeostasis Model Assessment.

\section{Conclusiones}

- Todos los índices aterogénicos en los pobladores adultos del distrito de Trujillo alcanzan mayoritariamente diagnóstico de riesgo cardiovascular, observándose con mayor prevalencia en el índice TG/cHDL, cercano al 75 \%; seguido por el índice de Castelli (CT/CHDL) y finalmente el índice de Kannel (cLDL/cHDL).

- Se ha evidenciado una alta prevalencia de obesidad abdominal, superando el $60 \%$ en los adultos trujillanos.

- Existe asociación muy significativa entre el índice TG/ cHDL y la presencia de obesidad abdominal. El índice TG/cHDL puede ser utilizado en pacientes obesos en su diagnóstico del perfil lipídico como valiosa información de seguimiento frente a una insulinorresistencia o riesgo de enfermedad cardiovascular

\section{REFERENCIAS}

1. Sánchez $A$, Muhn Ma, Lovera $M$, Ceballos B, Bonneau $\mathrm{G}$, Pedrozo W, et al. Índices antropométricos predicen riesgo cardiometabólico: Estudio de cohorte prospectivo en una población de empleados de hospitales públicos. RAEM. 2014;51(4):185-91

2. Instituto Nacional de Estadística e Informática-INEI. Perú: Enfermedades transmisibles y no transmisibles, 2019. Lima: INEI, 2020.

3. Bryce -Moncloa A, Alegría Valdivia E, San Martín -San Martín MG. Obesidad y riesgo cardiovascular. An Fac Med [Internet] 2017;78(2):202-206. [Consultado 2019 Nov 16] Disponible en: doi:10.15381/anales.v78i2.13218

4. Bohórquez- Rivero JJ, Rivera-Moreno MM, Lavalle- CM. Leptina y su Participación en la Enfermedad Arterial Coronaria Leptin and its Participation in the Coronary Artery Disease. [Internet] Archivos de Medicina 2020; 16(1):3-5. [Consultado 2019 Nov 16]. Disponible en: doi: $10.3823 / 1423$

5. Millán J, Pintó $X$, Muñoz $A$, Zúñiga $M$, Rubiés-Prat J, Pallardo LF, et al. Cocientes lipoproteicos: significado fisiológico y utilidad clínica de los índices aterogénicos en prevención cardiovascular. Clin Invest Arterioscl. [Internet]. 2010; 22(1):25-32. [Consultado 2019 Nov 16] Disponible en: doi:10.1016/S0214-9168(10)70005-X

6. Acevedo $M$, Krämer V, Tagle R, Corbalán R, Arnaíz P, Berríos $X$, et al. Total/HDL cholesterol ratio and non $\mathrm{HDL}$ cholesterol as predictors for increased intima media thickness. Total Cholest ratio non HDL Cholest as Predict increased intima media Thick. [Internet] 2012; 140(8):969-76 32 [Consultado 2019 Nov 16] Disponible en: doi: 10.1590/S0034-98872012000800001

7. Domingo FM. Utilidad de los índices aterogénicos como marcadores biológicos del síndrome metabólico [Tesis doctoral en Medicina] Madrid: Universidad Complutense de Madrid; 2012

8. Manninen V, Tenkanen L, Koskinen P, Huttunen JK, Mänttäri $M$, Heinonen $O P$, et al. Joint effects of serum triglyceride and LDL cholesterol and HDL cholesterol concentrations on coronary heart disease risk in the Helsinki Heart Study: Implications for treatment. Circulation [Internet] 1992; 85(1):37-45. [Consultado 2019 Nov 16] Disponible en: doi: 10.1161/01.cir.85.1.37

9. Soutelo J, Graffigna M, Honfi M, Migliano M, Aranguren $M$, Proietti A, et al. Índice triglicéridos/HDL-colesterol: en una población de adolescentes sin factores de riesgo cardiovascular. ALAN. 2012; 62(2): 167-171.

10. Singh SK, Aggarwal J, Kathariya G, Manzoor S. Usefulness of the TG/HDL ratio in predicting cardiovascular risk: A MMIMSR experience. JK Sci. 2020; 22(2): 75-9.

11. Gonzales R. Indicadores de riesgo aterogénico como predictores del síndrome metabólico en una población del Municipio Sifontes del Estado Bolívar Venezuela. SABER. Revista Multidisciplinaria del Consejo de Investigación de la Universidad de Oriente. 2016; 28 (2): 221-9.

12. Cuschieri S, Vassallo J, Calleja N, Pace N, Mamo J. The effect of age, gender, TG/HDL-C ratio and behavioral lifestyles on the metabolic syndrome in the high risk Mediterranean Island population of Malta. Diabetes Metab Syndr Clin Res Rev [Internet] 2017;11(2017):S3217. [Consultado 2019 Nov 16]. Disponible en: doi:10.1016/j.dsx.2017.03.009

13. Consenso Latinoamericano de la Asociación Latinoamericana de Diabetes (ALAD). Epidemiología, diagnostico, control, prevención y tratamiento del síndrome metabólico en adultos. Rev. Asoc. Latinoam. Diab. [Internet] 2010; 18(1):25-44[Consultado 2019 Ago 24] Disponible en: http://www.revistaalad.com/pdfs/100125-44.pdf

14. Asociación Médica Mundial. (AMM). Declaración de Helsinki de la AMM - Principios éticos para las investigaciones médicas en seres humanos. $64^{\mathrm{a}}$ Asamblea General; 2013 octubre. Fortaleza, Brasil: AMM [Internet] 2013 [Consultado 2019 Ago 2019] Disponible en: https://www.wma.net/es/policies-post/declaracionde-helsinki-de-la-amm-principios-eticos-para-lasinvestigaciones-medicas-en-seres-humanos/

15. Perú. Ley N.o 29733. Ley de Protección de datos personales. El Peruano (Jul. 3 2011)

16. Uruska A, Zozulinska-Ziolkiewicz, D, Niedzwiecki, P, Pietrzak, M, \&Wierusz-Wysocka, B. (2018).TG/HDL-C ratio and visceral adiposity index may be useful in assessment of insulin resistance in adults with type 1 diabetes in clinical practice. Journal of Clinical Lipidology, [Internet] 2018; 12(3): 734-740. [Consultado 2020 Nov 19] Disponible en: doi:10.1016/j.jacl.2018.01.005 
17. Coronel L. Índice aterogénico y factores de riesgo en diabéticos del programa de salud adulto mayor del Hospital General de Jaén 2019 [Tesis de licenciado tecnólogo médico] Jaén: Universidad Nacional de Jaén; 2019.

18. Castrillón-Lujan C. Estudio Exploratorio de la Asociación entre Índices Antropométricos (índice cintura estatura, índice cintura cadera e índice de masa corporal) y Componentes del Síndrome Metabólico [Tesis de maestría en Diabetes y Obesidad] Lima: Universidad Peruana Cayetano Heredia; 2018.

19. Vega-Robledo GB, Rico-Rosillo MG. Adipose tissue: Immune function and alterations caused by obesity. Rev Alerg Mex. [Internet] 2019; 66(3): 340-53 [Consultado 2020 Dic 09] Disponible en: doi: 10.29262/ram.v66i3.589

20. Suverza A, Haua K. Antropometría y composición corporal. El $A B C D$ de la evaluación del estado de nutrición. México: Mc Graw Hill; 2010.

21. Ascaso J, González Santos P, Hernández-Mijares A, Mangas-Rojas A, Masana L, Millán J, et al. Management of dyslipidemia in the metabolic syndrome. Recommendations of the Spanish HDL Forum. Am J Cardiovasc Drugs. [Internet] 2007; 7:39-58 [Consultado 2020 Dic 19] Disponible en: doi: 10.2165/00129784200707010-00004.

22. Berrocal N, Torres A. Relación entre Perfil lipídico e Índices aterogénicos con el Nivel de Hemoglobina Glicosilada en pacientes atendidos en el Hospital María Auxiliadora, 2017 [Tesis de licenciatura en tecnología médica en laboratorio clínico y anatomía patológica] Lima: Universidad Norbert Wiener; 2018.

23. Rosas A, Lama G, Llanos-Zavalaga F, Dunstan J. Prevalencia de obesidad e hipercolesterolemia en trabajadores de una institución estatal de Lima - Perú. Rev Peru Med Exp Salud Pública. 2002; 19 (2): 87-92.

24. Mawyin A, Saca D, Camargo C, Chávez B, Ludizaca D, Rodríguez $\mathrm{D}$, et al. HDL disfuncional en la diabetes mellitus tipo 2: una conexión entre la inflamación crónica y el riesgo cardiovascular. Revista Latinoamericana de Hipertension. 2018;13(3): 206-221.

25. Malik S, Budoff MJ, Katz R, Blumenthal RS, Bertoni AG, Nasir $\mathrm{K}$, et al. Impact of subclinical atherosclerosis on cardiovascular disease events in individuals with metabolic syndrome and diabetes: the multi-ethnic study of atherosclerosis. Diabetes Care. [Internet] 2011; 34(10): 2285-2290. [Consultado 2020 Oct 10] Disponible en: doi:10.2337/dc11-0816

26. Khazaál MS. Atherogenic Index of Plasma (AIP) As a Parameter in Predicting Cardiovascular Risk in Males Compared To the Conventional Dyslipidemic Indices (Cholesterol Ratios). J Med. [Internet] 2013;
6(1):1506-13 [Consultado 2020 Oct 10] Disponible en: PMID: 26793631

27. Roa $M$, Arata $G$, Valeri L, Velázquez E. Relación entre el cociente triglicéridos/cHDL, índices de resistencia a la insulina y factores de riesgo cardiometabólico en mujeres con síndrome del ovario poliquístico. Endocrinología y Nutrición [Internet], 2009, 56(2), 59-65 [Consultado 2020 Oct 10] Disponible en: doi:10.1016/ s1575-0922(09)70553-4

28. Delgado A, Valdés Y, Marcel E. Obesidad visceral: predictor de diabetes mellitus tipo 2 y enfermedades cardiovasculares. Rev Latinoam Patol Clínica y Med Lab. 2016; 63(2): 67-75.

29. Belén L, Oliva ML, Maffei L, Rossi ML, Squillace $C$, Alorda $\mathrm{MB}$, et al. Relación TG/HDL-C y resistencia a la insulina en mujeres adultas argentinas según su estado nutricional. Rev Española Nutr Humana y Dietética. 2013; 18(1): 18-24.

30. Von Bibra H, Saha S, Hapfelmeier A, Müller G,Schwarz P. Impact of the Triglyceride/High-Density Lipoprotein Cholesterol Ratio and the Hypertriglyceremic-Waist Phenotype to Predict the Metabolic Syndrome and Insulin Resistance. Horm Metab Res. 2017; 49(07):542-9. [Consultado 2020 Oct 10] Disponible en: doi: 10.1055/s0043-107782

31. Pérez $M$, Cuartas $S$. Diabetes tipo 2 y síndrome metabólico, utilidad del índice triglicéridos/HDL colesterol en Pediatría. Revista Cubana de Pediatría. 2016; 88(3): 335-47.

32. González-Chávez A, Simental-Mendía LE, ElizondoArgueta S. Elizondo-Argueta, Elevated triglycerides/ $\mathrm{HDL}$-cholesterol ratio associated with insulin resistance, Cir. Cir. 2011 [Internet] 2011; 79(2):126-31 [Consultado 2020 Oct 10] Disponible en: PMID: 21631973

33. Karelis AD, Pasternyk SM, Messier L, St-Pierre DH, Lavoie JM, Garrel D, Rabasa-Lhoret R. Relationship between insulin sensitivity and the triglyceride-HDL-C ratio in overweight and obese postmenopausal women: a MONET study, Appl. Physiol. Nutr. Metab. [Internet] 2007; 32(6):1089-96 [Consultado 2020 Oct 10] Disponible en: doi:10.1139/H07-095

34. Murguía-Romero $M$, Jiménez-Flores JR, Sigrist-Flores $S C$, Espinoza-Camacho MA, Jiménez-Morales $M$, Piña $E$, et al. Plasma triglyceride/HDL-cholesterol ratio, insulin resistance, and cardiometabolic risk in young adults. J Lipid Res. [Internet] 2013; 54(10): 2795-9. [Consultado 2020 Oct 10] Disponible en: doi:10.1194/jlr.M040584

35. Hernández-Vite $\mathrm{Y}$, Elizalde-Barrera $\mathrm{Cl}$, Flores Alcántara MG, Vargas-Ayala G, Loreto-Bernal ML. Asociación entre el índice triglicéridos/colesterol HDL y la glucosa alterada en ayuno en pacientes normotensos con obesidad y sobrepeso. Med Int Méx. 2015; 31: 507-515. 